\title{
Pertinencia del diseño curricular del Bachillerato Científico con Énfasis Ciencias Básicas de la Educación Media para la formación universitaria en las carreras de ingenierías
}

Modalidad: Proyectos Institucionales

Proponente: Universidad Iberoamericana (UNIBE) Ejecutor

Asociado: Ministerio de Educación y Cultura, Centro de Investigación e Innovación Educativa (CIIE).

Equipo Técnico Profesional

Responsable técnico: Graciela Molinas Santana, Coordinadora General del Proyecto de Investigación.

Equipo de investigadores:

- María Victoria Zavala (UNIBE).

- Rodolfo Elías (UNIBE).

- Hugo Speratti (UNIBE).

- Alcira Sosa (UNIBE).

- Rodrigo Gustavo Brítez (MEC).

- Sara López (MEC).

- Mariana Gomez (MEC).

- Daniel Felman, Universidad de Buenos Aires. (UBA), Asesor Internacional del proceso de investigación.

Área de la Propuesta: Disciplina Científico - Tecnológica relacionada al Proyecto Ciencias Sociales. Ciencias de la Educación

Síntesis del Proyecto

\section{Objetivos principales del proyecto}

La formación de los recursos humanos a través del Sistema Educativo Nacional es de vital relevancia para el desarrollo de las personas y del país, los niveles más comprometidos para la formación profesional son las del Nivel Medio y la Universidad. Las carreras de ingenierías en los últimos años se volvieron interesantes para los egresados de la Educación Media, quienes presentan dificultades significativas para demostrar los saberes mínimos para 
Pertinencia del diseño curricular del Bachillerato Científico con Énfasis Ciencias Básicas de la Educación Media para la formación universitaria en las carreras de ingenierías

el ingreso a dichas carreras. Esta situación Ileva a la pregunta central ¿cuál es la pertinencia del curriculum del Bachillerato científico con énfasis en Ciencias Básicas de la Educación Media en relación a los saberes exigidos para el ingreso en las carreras de ingenierías? Por cuya razón se pretende en esta investigación "Analizar la pertinencia del diseño curricular de la Educación Media- Bachillerato Científico con énfasis en Ciencias Básicas y Tecnología en relación a los saberes requeridos para la formación universitaria en las carreras de las Ingenierías.

\section{Objetivos específicos del proyecto}

1. Caracterizar los saberes mínimos necesarios para la formación universitaria integral en las carreras de las ingenierías.

2. Caracterizar la propuesta curricular del Bachillerato Científico con énfasis en Ciencias Básicas y Tecnología (Educación Media).

3. Determinar el nivel de desarrollo efectivo de los contenidos prescriptos en el área específica de Ciencias Básicas y su s tecnologías del curriculum de la Educación Media.

4. Identificar la relación entre los saberes requeridos para la formación universitaria en las carreras de Ingenierías y el currículum del Bachillerato Científico con énfasis en Ciencias Básicas y Tecnología (Educación Media).

5. Describir las experiencias vivenciadas por estudiantes ingresantes y docentes en las carreras de ingenierías en relación con los saberes adquiridos en el Bachillerato Científico con énfasis en Ciencias Básicas del Nivel de la Educación Media.

6. Describir las experiencias vivenciadas por estudiantes y docentes del Bachillerato Científico con énfasis en Ciencias Básicas del Nivel de la Educación Media en relación a los saberes adquiridos en el Nivel Medio.

7. Difundir la información generada en los resultados de la investigación.

\section{Resultados esperados de la investigación}

1. Mapeo de saberes requeridos para la formación universitaria en las carreras de las ingenierías.

2. Mapeo de saberes desarrollados en el Bacillerato Cientifico con énfasis en Ciencias Básicas, en la Educación Media.

3. Cuadro de relaciones entre la formación de la Educación Media y los saberes necesarios para la formación universitaria en las carreras de las ingenierías.

4. Informe de experiencias de estudiantes y docentes.

5. Informe final de investigación. Publicación y difusión. 


\section{Proyectos de la UNIBE}

\section{EsPeCIFICACIÓn del ProyeCto}

\section{Antecedentes de la Propuesta. Marco teórico y conceptual de partida}

"La educación, se concibe como uno de los principios fundamentales para el desarrollo de un país, ya que una persona adecuadamente formada, es una persona que puede contribuir al progreso del país, para ello, su formación debe responder a las necesidades específicas del mercado laboral, las cuales cambian con el tiempo, es por ello que se vuelve esencial realizar constantes revisiones de las propuestas educativas propiciadas por el sistema educativo."

El Sistema Educativo Nacional (SEN) se organiza en cuatro niveles, que a su vez se divide en ciclos y años. El estudio propuesto focalizará la Educación Media (EM), nivel que se desarrolla posterior a la Educación Escolar Básica, se compone de tres años curso, de carácter obligatorio y gratuito desde el 2012. El proceso de reforma curricular impulsada desde 1993 en todo el SEN incide en la EM desde año 2002. Esto quiere decir que el actual bachillerato era selectivo, la mayoría de los estudiantes está fuera del sistema (MEC.2010) y quienes permanecen en él, no aprenden lo básico en áreas como Matemática y Lengua. Los estudiantes egresan con limitaciones importantes para operar con computadoras, pensar reflexiva y críticamente e interpretar ideas y mensajes. Los resultados de las evaluaciones y de los estudios concluyen que no se encuentra correspondencia entre las habilidades que se desarrollan en la institución escolar y los requerimientos fuera de ese ámbito, tanto en la vida laboral como para los estudios superiores.

La preparación para la Educación Superior Universitaria, en tanto que puede analizarse en términos de cursos o áreas temáticas abordadas en la Educación Secundaria y rendimiento educativo de los estudiantes de acuerdo a pruebas nacionales estandarizadas no se limita a los contenidos curriculares, pero las posibilidades de adquirir lo que David T. Conley (2007) indica son una serie capacidades cognitivas y metacognitivas que pueden ser igual o más importantes que el contenido de conocimientos brindados en los colegios o cursos preparatorios de ingreso a la universidad.

El estudio abordará el Bachillerato Científico con énfasis en Ciencias Básicas y sus Tecnologías, buscará observar la relación que hay entre el diseño curricular de la Educación Media y la preparación para la universidad, sobre todo con las carreras afines. La mirada más profunda se colocará sobre el área de ciencias Básicas y sus Tecnologías del plan común (Ciencias Naturales y Salud, Física y Química) y las ciencias y Tecnología del plan específico (Química, Física, Biología, Lógica Matemática y Estadísticas, Geología, Educación Ambiental y Salud) y en el nivel de la Educación Superior, en las áreas

La manera de concebir el curriculum pasa por una revisión de concepciones clásicas hasta llegar a las que se consideran las más actuales, como las presentadas por Hilda Taba (1962) como un instrumento que expresa una toma de decisiones ordenadas que afectan la selección y organización del contenido, 
Pertinencia del diseño curricular del Bachillerato Científico con Énfasis Ciencias Básicas de la Educación Media para la formación universitaria en las carreras de ingenierías

la elección de experiencias de aprendizaje y los planes para lograr condiciones óptimas para que se produzca el aprendizaje; por su parte Stenhouse( 1987) plantea que es un medio con el cual se hace públicamente disponible la experiencia consistente en intentar poner en práctica una propuesta educativa. Con una posición más social, Jurjo Torres,( 2002) lo describe como un proyecto educativo que se planifica y desarrolla a partir de una selección de la cultura y de las experiencias en las que se desea que participen las nuevas generaciones con el fin de socializarlas y capacitarlas para ser ciudadanos y ciudadanas solidarias, responsables y democráticas". Lo común de las concepciones presentadas remiten a la idea de selección de saberes considerados válidos para el aprendizaje, que orientan las acciones pedagógicas en los centros educativos, dichos saberes se explicitan en "instrumentos oficiales que se consideren legítimos y principales a través del cual (o de los cuales), en cada país o región, el Estado ordena las prácticas de enseñanza y determina cual es el saber oficializado" (Gvirtz y Palamidessi,: 2004: 75).

\section{Bibliografía relacionada}

Los siguientes textos citados permitirán profundizar en lo referente al curriculum, concepciones discusiones y podrán contextualizar el posicionamiento de la perspectiva con la que se analizará la información a ser obtenida

Gimeno Sacristán, José (1991). El curriculum: Una reflexión sobre la práctica. Madrid: Morata.

Palamidessi, Mariano Y Gvirtz, Silvina (2006). El ABC de la tarea docente: currículum y enseñanza. Buenos Aires: Aique.

Stenhouse, Laurence (1993). La investigación como base de la enseñanza. Madrid: Morata.

Zabalza, Miguel A. (2000). Diseño y desarrollo curricular. Madrid: Narcea.

Arnaz, José. (2004). La Planeación Curricular. México: Trillas

Los siguientes autores permitirán ahondar en las referencias basadas en investigaciones sobre los retos de la preparación para la universidad, concepción de la universidad y el abordaje de la transición entre la secundaria y la universidad.

Juré y Solari.(2006). El espacio de competencias en la articulación curricular por disciplinas entre el nivel de medio y universitario. $1^{a}$ Río Cuarto: Universidad Río Cuarto, Conley, D. T. (2007). Redefining College Readiness. Educational Policy Improvement Center.; (2007).Toward a More Comprehensive Conception of College Readiness. Eugene, OR: Educational Policy Improvement Center, University of Oregon.

Conley, D. T. (2008). Rethinking College Readiness. New Directions for Higher Education, 144:3-13

Conley, D. T. (2007). The challenge of College Readiness. Educational Leadership, 64(7), 23. 


\section{Proyectos de la UNIBE}

McAlister, S., \& Mevs, P. (2012). College Readiness: A Guide to the Field. Annenberg Institute for School Reform at Brown University.

Roderick, M., J. Nagaoka, and V. Coca. 2009. College Readiness for All: The Challenge for

Urban High Schools. The Future of Children, 19,no. 1:185-210.

Long, M. C., latarola, P., \& Conger, D. (2009). Explaining gaps in readiness for college-level math: The role of high school courses. Education, 4(1), 1-33.

Martinez, M., \& Klopott, S. (2005). The link between high school reform and college access and success for low-income and minority youth. American Youth Policy Forum.

Byrd, K. L., \& MacDonald, G. (2005). Defining college readiness from the inside out: First-generation college student perspectives. Community College Review, 33(1), 22-37.

Kirst, M., \& Venezia, A. (2001). Bridging the great divide between secondary schools and postsecondary education. Phi Delta Kappan, 83(1), 92-97.

Barnett, E. A., Fay, M. P., Trimble, M. J., \& Pheatt, L. (2013). Reshaping the college transition: early college readiness assessments and transition curricula in four states.

Venezia, A., \& Jaeger, L. (2013). Transitions from High School to College. The Future of Children, 23(1), 117-136.

\section{Metodología de Investigación}

El diseño de investigación propuesto es: no experimental de naturaleza descriptiva, asumiendo un enfoque mixto (cualitativo y cuantitativo) para la recolección de los datos y su posterior análisis. Se tendrán en cuenta dimensiones, categorías e indicadores como modelo inicial pudiendo ser ajustado a partir de los datos obtenidos en el trabajo de campo y el análisis de los documentos curriculares.

En la primera etapa: se realizará análisis curricular, a través de la técnica de observación documental los documentos curriculares de la Educación Media y los programas de ingreso para las carreras de ingenierías teniendo como base las categorías e indicadores predefinidos. El método de análisis parte de la organización, categorización y codificación de los datos para describirlas e interpretarlas de manera preliminar.

En la segunda etapa se aplicara la técnica de entrevista en profundidad, por un lado, a los profesores de las universidades seleccionadas (tres del interior y tres de la capital) y por el otro lado, a los docentes que se desempeñan en el tercer año de la Educación Media, en las áreas focalizadas.

En la tercera etapa se recurrirá a recoger datos de los estudiantes para lo cual se aplicaran encuestas y Focus group, por un lado, a estudiantes de los 
Pertinencia del diseño curricular del Bachillerato Científico con Énfasis Ciencias Básicas de la Educación Media para la formación universitaria en las carreras de ingenierías

primeros años de las carreras de ingenierías y por el otro lado, a estudiantes del último año de los Bachillerato Científico con énfasis en Ciencias Básicas y Tecnología (Educación Media) de los colegios seleccionados para el estudio.

Procesamiento de los datos, en las etapas 2 y 3, los datos obtenidos en las entrevistas y focus groups se transcribirán a texto, y se tratará en dos niveles. En el primer nivel se generarán unidades de significado, se comparan esas unidades a fin de crear categorías y en el segundo nivel se comparan las categorías creadas a fin de interpretar los datos y desarrollar patrones que permitan construir los mapas y gráficos de relaciones. Los datos de la encuestas serán organizados y procesados en planillas Excel para identificar datos significativos que permitan triangularlos con los remitidos por las otras técnicas aplicadas.

Las instituciones a ser seleccionada para la etapa 1 y 2, deberán deberá reunir los siguientes criterios: universidades que ofrecen carreras de Ingenierías, tres del interior del país y tres de la capital y central, que realizan evaluaciones de ingreso, que ofrezcan la carrera en los últimos 5 años( 2009 al 2013) ; instituciones que imparten la Educación Media ofreciendo el Bachillerato Científico con énfasis en Ciencias Básicas y Tecnología, que cuenten con egresados en los últimos 5 años, de gestión pública, privada y subvencionada, que se encuentren en la capital y en dos departamentos del interior del país, del área de influencia de las universidades seleccionadas para el estudio.

La población seleccionada para la etapa 2 y 3 lo constituirán estudiantes y docentes de las instituciones seleccionadas. La muestra a ser aplicada será la no probabilística y estratificada; la cantidad será el resultado de los 30\% de la población total. Para la aplicación de la encuesta se propone recoger el $50 \%$ de la población total de la población de estudiantes de la Educación Media e ingresantes en las carreras de ingenierías. La cantidad focus groups será definida por el $20 \%$ del total de estudiantes, divido por la cantidad de participantes teniendo en cuenta el rango de validez de la técnica

Los resultados obtenidos de la Educación Media (Bachillerato científico con énfasis en Ciencias Básicas) y las carreras de ingenierías permitirán la construcción de la relación de elementos que identifiquen la pertinencia o la incongruencia. Además se tendrá en cuenta la dimensión ética de la investigación en todo el proceso con el aseguramiento del anonimato de los participantes, el secreto de los datos particulares obtenidos de los informantes y el cuidado de enmarcarse en teorías curriculares para emitir juicios sobre los diseños curriculares analizados. 


\section{Proyectos de la UNIBE}

\section{RELEVANCIA DE LA PROPUESTA DE INVESTIGACIÓN}

Se describe la trascendencia del tema de investigación en tres dimensiones: Científica, Social y Contemporánea.

Relevancia para orientar políticas educativas: Contar con un análisis del curriculum del Bachillerato Científico con énfasis en Ciencias Básicas permita develar la pertinencia o incongruencia del ámbito propedéutico del mencionado bachillerato en relación a las demandas de la universidad (en carreras de ingenierías). Los resultados servirán de base para tomar decisiones sobre políticas pertinentes para incorporar innovaciones curricular, así como para definir el diseño curricular consensuado para el periodo de nivelación de saberes en estudiantes que pretenden ingresar en las carreras de ingenierías, asumiendo que las mismas definirán el desempeño eficiente y eficaz del trayecto académico en dichas carreras.

Relevancia (social): El resultado de la investigación incorpora la voz de los estudiantes y docentes de ambos niveles, EM y Educación Superior, reportando sus percepciones desde la experiencia misma de la acción educativa. Además permitirá poner en la agenda social el debate sobre la articulación de dos niveles del Sistema Educativo paraguayo muy relevantes para la formación de recursos humanos del país

Relevancia Científica: El abordar esta investigación aportaría a contar con información científica local, ante la falta de artículos científicos sobre tema. También la propuesta metodológica planteada, desarrollada y validada podrá ser utilizada en otros estudios sobre pertinencia curricular. Además, se considera que la participación de investigadores del MEC, así como de la UNIBE en este proceso dejará capacidad instalada y la consolidación del perfil de investigadores en la línea de estudio curricular.

\section{Descripción del Plan de Trabajo}

Detalle el tiempo de duración del proyecto (a partir de la firma del contrato)

La duración de la investigación se proyecta para dos años (24 meses), considerando que se aplicarán técnicas e instrumentos cualitativos con una población muy dispersa y el proceso de análisis deberá contar con validaciones de asesores y experto para lograr confiabilidad.

\section{Los principales resultados}

Primera etapa: Recopilación de datos y mapeo de saberes requeridos para la formación universitaria en las carreras de las ingenierías

Segunda etapa: Recopilación de datos y mapeo de saberes desarrollados en el Bachillerato Científico en las Ciencias Básicas en la EM.

Tercera etapa: Categorización de experiencias de estudiantes y docentes consultados 
Pertinencia del diseño curricular del Bachillerato Científico con Énfasis Ciencias Básicas de la Educación Media para la formación universitaria en las carreras de ingenierías

Cuarta etapa: Elaboración del cuadro de relaciones .

Quinta etapa: Informe de experiencias de estudiantes y docentes

Secta etapa: Elaboración del informe final, presentación y difusión

\section{ESTRATEGIA DE LA COMUNICACIÓN}

Se describa los mecanismos de transferencia, validación, difusión y/o diseminación del conocimiento que el proyecto plantea

La estrategia de comunicación se centrara en presentación de los avances de la investigación y el informe final a través de foros para el análisis y la generación de debates en relación al tema abordado; documentos impresos a presentar a los actores relevantes que están involucrados en la investigación; la elaboración de artículo científico a ser publicado en revistas científicas y plataforma virtual de la universidad Iberoamericana y la del MEC. 